
\title{
Research Suare \\ Stretchable Conductive Materials Based On Braided Cnt Yarns On Elastomeric Core
}

\author{
Avia J Bar ( $\square$ Avia_Bar@student.uml.edu ) \\ University of Massachusetts Lowell \\ Joey Mead \\ University of Massachusetts Lowell \\ Hanna Dodiuk \\ Shenkar College of Engineering and Design Ramat-Gen \\ Samuel Kenig \\ Shenkar College of Engineering and Design Ramat-Gen
}

\section{Research Article}

Keywords: Composites, Stretchable conductive, Braiding, Braid angle, Elastomer, CNTYs, Mechanical behavior

Posted Date: January 28th, 2022

DOI: https://doi.org/10.21203/rs.3.rs-1292460/v1

License: (c) (1) This work is licensed under a Creative Commons Attribution 4.0 International License. Read Full License 


\title{
STRETCHABLE CONDUCTIVE MATERIALS BASED ON BRAIDED CNT YARNS ON ELASTOMERIC CORE
}

\author{
Corresponding author: Avia J Bar ${ }^{1}$, Avia_Bar@student.uml.edu \\ Joey Mead ${ }^{1}$, Hanna Dodiuk², Samuel Kenig ${ }^{2}$ \\ ${ }^{1}$ Department of Plastics Engineering, University of Massachusetts Lowell, USA \\ ${ }^{2}$ Department of Polymers and Plastics Engineering, Shenkar College of Engineering and Design \\ Ramat-Gen, Israel
}




\begin{abstract}
The mechanical properties of braided carbon nanotube yarns (CNTYs) on an elastomeric core to produce stretchable conductive materials were modeled and studied under tension. The elastomeric core served as the stretchable spring and the CNTYs braiding, with shape changing capabilities, as the conductive shell. The model predicts the stress-strain behavior of the composite as a function of the initial braiding angle and the number of pitches. The innovative aspect was included in the model related to the friction between the braid and the core. Results indicated good agreement between the theoretical model and the experimental results. Since the rate of the diameter decrease of the CNTYs braid was higher than that of the elastomeric core diameter, squeezing out of the core through the braid inter yarn space occurred. This limited the maximum potential extension of the braid. Thus, a critical strain was defined where the braid came into contact with the core. The addition of the friction stresses made a significant contribution to the overall stresses and the accuracy of the model and its agreement with the experimental results. An apparent friction coefficient was proposed to account for the effect of the elastomer core/braid interactive restriction and squeezing out of the elastomer through the braiding, as observed in experimental results. As the CNTYs are conductive, a stretchable conductive composite was obtained having a resistivity of $9.05 \times 10^{-4} \mathrm{Ohm} * \mathrm{~cm}$, which remained constant throughout the tensile loading until failure and under cyclic loading.
\end{abstract}

Keywords: Composites, Stretchable conductive, Braiding, Braid angle, Elastomer, CNTYs, Mechanical behavior 


\section{Introduction}

Stretchable electronics are comprised of deformable substrates and conductive elements that enable electronic devices with large deformations. These devices have two main challenges (1) achieving high constant conductivity under large deformation and (2) returning to the original position without a set ${ }^{1}$. There are many applications where stretchable electronics are of importance, such as health monitors, medical implants, artificial skins, human-machine interfaces, wearable internet of things, etc. $2,3,4,5,6,7,8$. Stretchability is defined as the ability of the conductive elements to be stretched reversibly in response to an applied force ${ }^{2}$. Stretchability can be achieved using one of the following approaches: a geometrically-based approach in which material designs are created that can enable large deformation or a materials-based approach in which the materials intrinsically stretch ${ }^{2,3}$. A geometrically-based approach is usually comprised of a non-stretchable conductive material and unique geometrical structures or architectures, such as out-of-plane wrinkles, serpentines, etc., that are stretchable. Usually, this technique requires sophisticated structural designs ${ }^{9}$. The material-based approach usually uses intrinsically stretchable conductive elastomers (rubbery electronics) ${ }^{3}$. One of the main drawbacks of this approach is that conductivity usually decreases during the stretch, but the materials have good stretchability ${ }^{2,10}$.

This work is based on combining the two approaches ${ }^{2,10}$ by developing composite materials for stretchable conductive electronics. The composites are based on braiding carbon nanotube yarns (CNTYs) on an elastomeric core to maintain high conductivity under large deformations with a low permanent set. This approach combines both geometrical and materials elements where the conductive braided structure can be extended to large deformations and maintain high electrical conductivity, while the elastomer core acts as an elastic spring for repeated stretching. A model was developed to understand the combined mechanical behavior of the composite structure. 
Tubular braided structures are found in various applications such as ropes, composite materials ${ }^{11}$, medical devices ${ }^{12}$, etc. ${ }^{13}$. Braiding geometries can be classified as diamond (one on one repeat), regular (two on two repeats), and Hercules (three on three repeats) based on the weave pattern. The tubular structure prepared by an ancient technique is called circular braiding, which involves at least three yarns. Each strand forms a well-defined weave pattern following a helical path.

Tubular braids consisting of an elastic core were investigated in 1978 by Phoenix ${ }^{14}$ and then by other researchers ${ }^{15,16,17}$. This unique structure has a unique combination of linear and nonlinear mechanical behavior during stretching ${ }^{18}$. The braid on core composite structure leads to large elastic deformations even for low stretch yarns.

Phoenix ${ }^{14}$ carried out pioneering work on the mechanical behavior of braid-core structures. He developed a model that described the linear response of a structure with a diamond braid. The model assumed small displacements, taking into account the undulation or crimp. The model's prediction of the modulus of elasticity was is in good agreement with the experimental results. Several parameters were found to affect the mechanical response, including the moduli of the core and fiber, Poisson's ratio of the core, the braid angle, and the strand crossovers' crimp angle. Later, Abbott ${ }^{15}$ expanded Phoenix's model by considering the non-linearity of the helically wrapped tape material and the elastic core but did not consider the waviness which is formed during the braiding process.

More than a decade later, Hopper ${ }^{16}$ proposed a model based on four modes. Each mode described the behavior of the braid and the elastic core and their relationship. For modes were used to describe the behavior. Modes I and IV described the tensile response of the elastic core, assuming that the braid and the elastic core were not in contact. Modes II and III described the behavior when the core and braid were in contact. This investigation was primarily theoretical without any experimental validation. 
The most recent model was formulated by Rawal ${ }^{19}$, describing the true stress for both the braid and the core, which in this case consisted of monofilament strands of low twists forming a multi-filament yarn. They obtained good agreement between the theoretical and experimental stress-strain curves. However, for yarns with relatively high stiffness, significant deviations were obtained due to the large radial stresses, which led to the core "squeeze out" between the yarns. The phenomenon of "squeezing out" resulted in a jammed structure where the braiding angle could not be easily decreased and inhibiting further stretching.

While these models are fairly complex, they do not include the effect of friction between the core and braid, which may significantly effect the mechanical behavior prior to squeeze out. Thus, the current work's primary objective was to model and study the tensile behavior of composite-based stiff yarns braided on an elastomeric core by also taking into account the friction stresses between the braided yarn and the core. The resultant model showed good agreement with the experimental results. Furthermore, an apparent friction coefficient was specified to account for the effect of the squeeze-out phenomena and the changes in the friction during the stretch.

\section{Model Development and Results}

Three ranges were included in the modeling. In range I, the core diameter was smaller than the braid's diameter, and therefore, there is no contact between the braid and core. In range II the core and the braid are in contact resulting in friction and normal stresses. In range II, the friction between the core and braided structure, the change in the core's diameter due to radial compression of the braid, and waviness are considered. Last, in range III, the core begins to squeeze out, and the fiber structure becomes jammed (Table 1). 
Table 1: Three Ranges For The Proposed Model

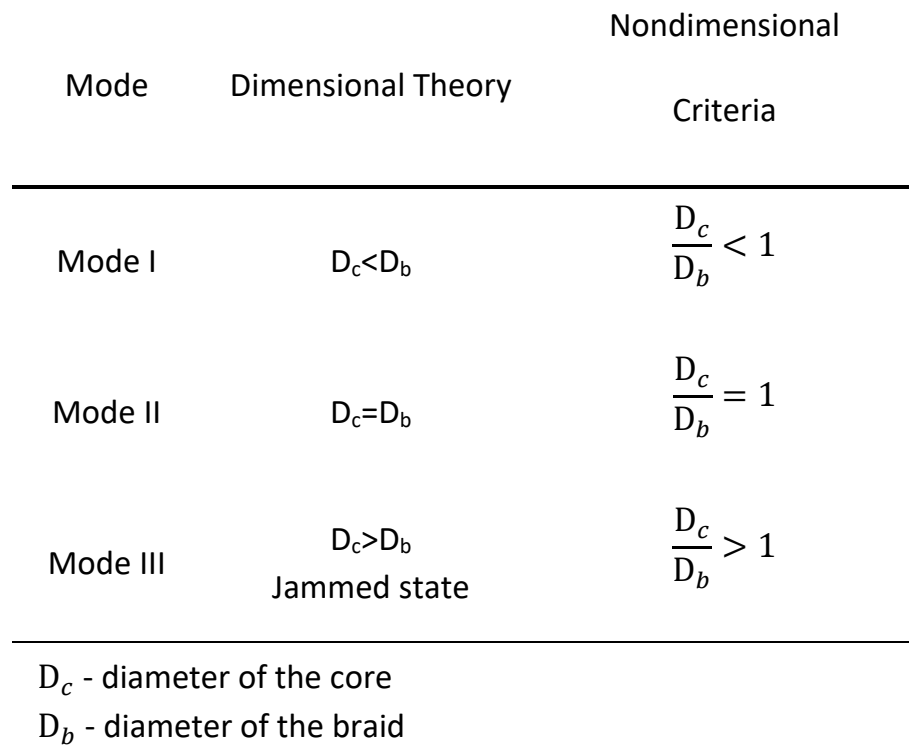

\section{Model Assumptions}

The model postulates a basic tubular braid geometry on an elastic core with the following assumptions:

- The braiding has an open braiding structure.

- Each yarn is assumed to follow a helical path, but locally the strand centerline follows an undulated path.

- The cross-sectional shape and diameter of the yarn remain unaffected during the application of tensile load.

- The core has a circular cross-section and is an isotropic material such that the volume of the core remains constant $(V \approx 0.5)$. 
- The radial and tangential strains exhibited by the core are assumed to be equivalent based on the assumption of symmetry.

- The jamming of the yarns cannot be neglected.

- The yarns are assumed to generate uniform stress on the core, which is balanced by the radial stress at its outer surface.

- The yarn is linearly elastic with modulus $\mathrm{E}_{0}$.

For each range there are additional assumptions as following, Range I assumed yarn strain is zero and the initial compression of the yarn on the core is small. Range II assumed a small displacement occurs in the yarn, the volume of the braided structure is conserved under tension and the core is assumed to be subjected to uniform radial compression.

\section{Analysis}

\section{Range I:}

Since there is no contact between the core and the braid ${ }^{19}$, the stress is a result of the true stress of the core $\left(\sigma_{c}\right)$ as in equation 1.1

$$
\sigma_{c}=\frac{F_{c}}{A_{c}}=\frac{4 F_{c}}{\pi D^{2}}
$$

Where, $F_{c}, A_{c}$ are the force acting on the core and the cross-sectional area of the core, respectively. $\mathrm{D}$ is the core diameter under a defined level of axial strain, assuming that it is an isotropic material. Therefore, the radial strain $\left(\varepsilon_{r}\right)$ and the axial strain $\left(\varepsilon_{z}\right)$ are related as in equation $1.2^{19}$ : 


$$
\left(1+\varepsilon_{z}\right)\left(1+\varepsilon_{r}\right)^{2}=1
$$

The core diameter and the radial strain are related as follows:

$$
D=D_{0}\left(1+\varepsilon_{r}\right)
$$

Where $D_{0}$ is the core diameters at zero axial strain and $\varepsilon_{r}$ is the radial strain. Using equation 1.2 and 1.3, the diameter of the elastomer core is obtained as:

$$
\frac{D^{2}}{D_{0}^{2}}=\frac{1}{\left(1+\varepsilon_{z}\right)}
$$

The diameter for the braid (d) is determined using equation 1.5 and based on geometric relations as shown in Figure 1.
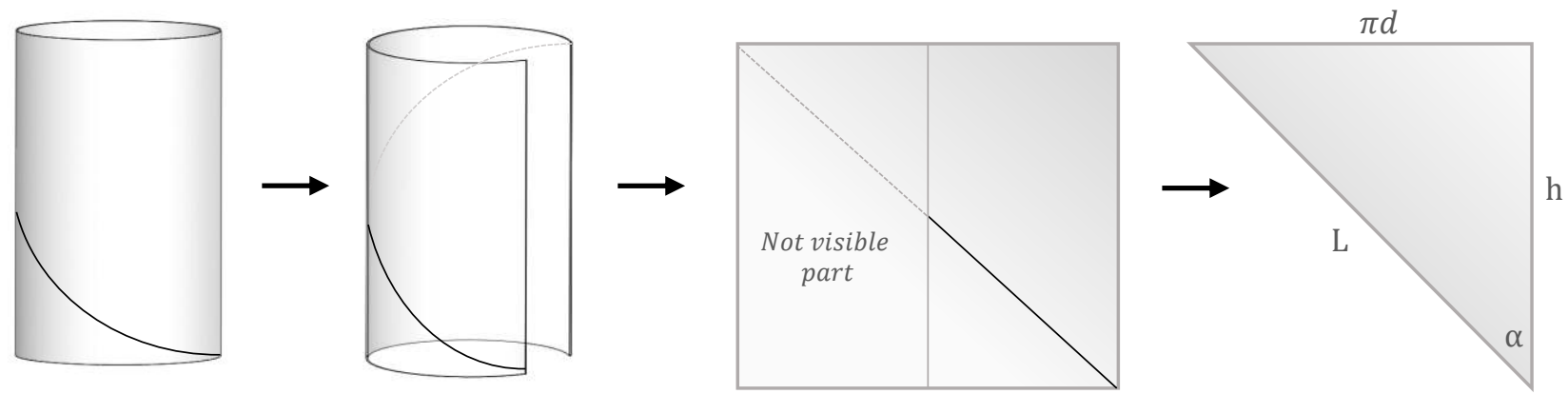

Figure 1. Geometric relations for a helical braid.

$$
\frac{\mathrm{d}}{d_{0}}=\frac{1}{\tan \left(\alpha_{i}\right)}\left(\frac{1}{\cos ^{2}\left(\alpha_{i}\right)}-\left(1+\varepsilon_{z}\right)^{2}\right)^{0.5}
$$


where $d_{0}$ is the initial braiding diameter and $\alpha_{i}$ is the initial braiding angle. The core and braid diameters, according to equations 1.3 and 1.5, are plotted in Figure 2 for different initial braiding angles.

The calculated results (Figure 2) show that the rate of change of the braid diameter and the core diameter are different. As seen in Figure 2, the curves intersect at different strains depending on the initial braiding angle. When the braid touches the core (intersection point), the material shifts from the behavior modeled by range 1 into the model for range 2 . At this point there are compression stresses on the core by the yarns.

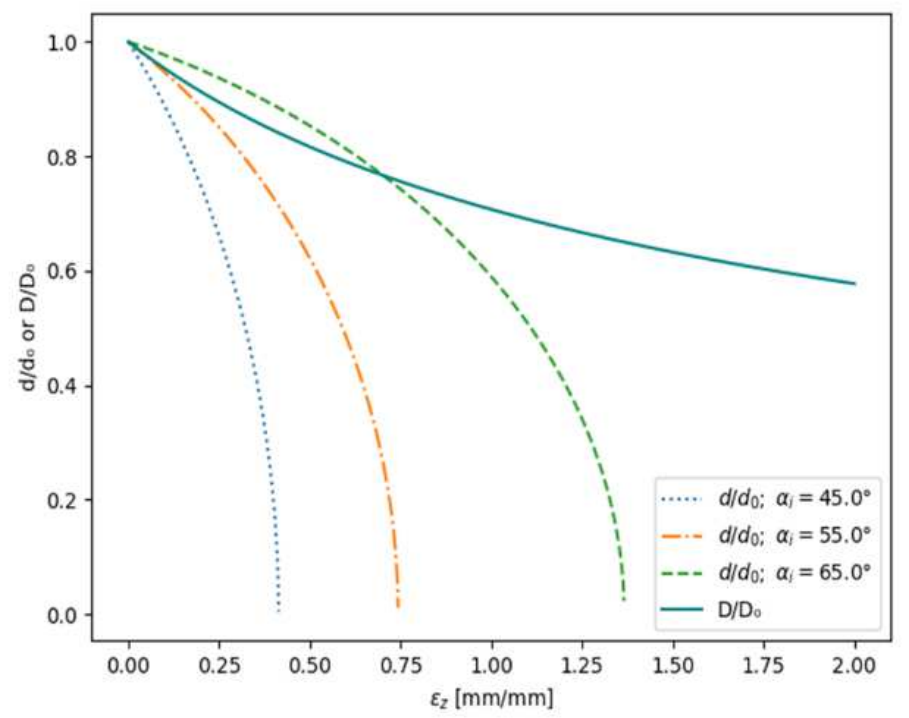

Figure 2. Variation of core diameter and braid diameter with different initial braiding angles as a function of axial strain.

For braiding angles lower than $55^{\circ}$ the curves intersect at small axial strains. For braiding angles above $55^{\circ}$, the intersection occurs at larger axial strains. After reaching the intersect point, the core will be compressed by the yarns and the rubber will squeeze out through the braid. 
Following contact between the braid and the core, the change in the braid angle involves the simultaneous change of the core and braid diameter, with the core being the limiting material. In this range friction between the yarn and the core is important and is described below for range II. As the braid angle decreases, one may approach the point where contact of the braid and core occur at very low strains and nearly immediate locking of the yarns due to squeeze out. With no possibility for a further change of the braid angle this leads to the third range where the yarns are strained elastically to failure.

The locking strain can be determined from Figure 2 from the asymptotes for each initial braiding angle. The value of maximum strain can be determined by calculating the vertical asymptote for each curve using equation 1.5 to obtain equation 1.6

$$
\lim _{\frac{d}{d_{0}} \rightarrow 0} \frac{d}{d_{0}}=\frac{1}{\tan \left(\alpha_{i}\right)}\left(\frac{1}{\cos ^{2}\left(\alpha_{i}\right)}-\left(1+\varepsilon_{z}\right)^{2}\right)^{0.5}
$$

Therefore, the maximum strain for each initial braiding angle is

$$
\varepsilon_{z, \max }=\frac{1}{\left|\cos \left(\alpha_{i}\right)\right|}-1
$$

\section{Range II:}

At a specific value of axial strain, the core and braid diameter equalize. At this point, the stress will be determined by contributions of the core and the braid. The tensile stress for the braid-core system is given by: 


$$
\sigma=\frac{F}{A_{b c}}
$$

Where $\mathrm{F}$ is the total axial force acting on the braid-core system. The force is comprised of three components: 1 . The axial force to extend the braid $\left(F_{b}\right) ; 2$. The axial force to elongate the core where the core itself is also under radial pressure from the braid $\left(F_{c}\right)$; and 3 . The axial friction force between the core and the braid $\left(F_{k}\right) \cdot A_{b c}$ is the total cross-sectional area of the tubular braid $\left(A_{b}\right)$ and the core $\left(A_{c}\right)$. Equation (2.2) and (2.3) describe the total axial force and cross-sectional area, respectively:

$$
\begin{gathered}
F=F_{b}+F_{c}+F_{k} \\
A_{b c}=A_{b}+A_{c}
\end{gathered}
$$

The axial force component of the braid is the total axial force on the filaments, which is equal to ${ }^{15}$ :

$$
F_{b}=N \varphi_{y} F_{f} \cos (\alpha)
$$

Where $F_{f}$ is the filament tension, $\mathrm{N}$ the number of the filaments and $\varphi_{y}$ is the yarn packing factor defined as the ratio of the volume of constituent filaments to the yarn volume. For the yarn tension, the axial force is ${ }^{19}$ :

$$
F_{f}=f\left(\varepsilon_{f}\right)
$$

Where, $\varepsilon_{f}$ is the yarn strain. For elastic behavior, the strain of the yarn was assumed to follow Hookean behavior (equation 2.6) 


$$
\sigma_{f}=E_{f} \varepsilon_{f}
$$

Therefore, the stress of the yarn can be obtained directly from the stress-strain curve ${ }^{20}$. From the geometry relation of the braid, the following equation was obtained:

$$
\left(\varepsilon_{z}+1\right)^{2}=\left(\varepsilon_{f}+1\right)^{2} \sec ^{2}\left(\alpha_{i}\right)-\left(\varepsilon_{r}+1\right)^{2} \tan ^{2}\left(\alpha_{i}\right)
$$

The axial strain and the filament strain can be related to each other by combining equations 1.2 2.7, which stem from the geometrical analysis of a helical wrapping. Thus, we can obtain the yarn strain according to 21,22 ,

$$
\varepsilon_{f}=\left[\left(1+\varepsilon_{z}\right)^{2} \cos ^{2}\left(\alpha_{i}\right)+\frac{\sin ^{2}\left(\alpha_{i}\right)}{\left(1+\varepsilon_{z}\right)}\right]^{0.5}-1
$$

The axial load in the elastic core $\left(F_{c}\right)$ is calculated based on the fact that the radial and circumferential strains are equivalent ${ }^{16}$, therefore:

$$
F_{c}=A_{c} E_{c} \frac{\left[(1-v) \varepsilon_{z}+2 v \varepsilon_{r}\right]}{(1-2 v)(1+v)}
$$

Where, $E_{c}$ is the tensile modulus of the core, $v$, is the Poisson's ratio of the core, which decreases as the axial strain $\left(\varepsilon_{z}\right)$ increases. By using equation (1.2) we obtain

$$
v=\frac{-\varepsilon_{r}}{\varepsilon_{z}}=\frac{\sqrt{\varepsilon_{z}+1}-1}{\varepsilon_{z} \sqrt{\varepsilon_{z}+1}}
$$


The radial strain $\left(\varepsilon_{r}\right)$ is influenced in this range by the compression stresses from the braid; therefore, elasticity should be taken into consideration as shown in equation $2.11^{19}$

$$
\varepsilon_{r}=-\frac{(1-2 v)(1+v)}{E_{c}} P_{R}-v \varepsilon_{z}
$$

Where, $P_{R}$ is the pressure (compression stress) on the core. The hoop tension of the filament $F_{f} \sin ^{2}(\alpha)$ will act over the helical turns per unit axial length $(1 / T)$, as expressed in the following equation ${ }^{14,15}$

$$
P_{R}=\frac{T F_{f} \sin (\alpha)}{R}=\frac{F_{f} \sin ^{2}(\alpha)}{2 \pi R^{2} \cos (\alpha)}
$$

Where, $R$ is the radius of the core and $\alpha$ is the braiding angle. Combining equations (2.11) and (2.12), we obtain

$$
\varepsilon_{r}=-\frac{4(1-2 v)(1+v) F_{f} N \sin ^{2}(\alpha)}{E_{c} 2 \pi D^{2} \cos (\alpha)}-v \varepsilon_{z}
$$

From equations (2.9) and (2.13), the axial load in the elastic core $\left(F_{c}\right)$ is given by

$$
F_{c}=A_{c}\left(E_{c} \varepsilon_{z}-\frac{N F_{f} v \sin ^{2}(\alpha)}{\pi R^{2} \cos (\alpha)}\right)
$$

The force component of the friction force $\left(\boldsymbol{F}_{\boldsymbol{k}}\right)$, in the axial direction, is obtained by summing the axial stress component of the yarns shown in equation 2.15 


$$
F_{k}=N \mu_{k} F_{N} \cos (\alpha)
$$

Where, $\mu_{k}$ is the coefficient of kinetic friction and $F_{N}$ is the normal force that acts on the core. The normal force is equal to the radial pressure $P_{R}$ multiplied by the contact surface area between the core and braid taking into account the number of pitches. The number of pitches, $N_{p}$ is defined by equation 2.6:

$$
\mathrm{N}_{\mathrm{P}}=\frac{\mathrm{L}_{\mathrm{s}}}{\tan \left(\alpha_{i}\right) \pi D_{S}}
$$

Where $D_{S}$ is the initial diameter of the braid and $\mathrm{L}_{\mathrm{S}}$ is the length of the braid on core sample. Therefore, the normal force, $F_{N}$, is

$$
F_{N}=\frac{2 F_{f} \sin ^{2}(\alpha)}{\pi D^{2} \cos (\alpha)} * \mathrm{~N}_{\mathrm{P}} * \frac{\pi D_{b} d_{f}}{\sin (\alpha)}=\frac{2 \mathrm{~N}_{\mathrm{P}} F_{f} \sin (\alpha) \times D_{b} \times d_{f} \times \pi \times\left(1+\epsilon_{z}\right)}{D^{2} \times \tan (\alpha)}
$$

Where $D_{b}$ is the braid diameter. Hence, as the radial pressure on the core increases, the friction force becomes more significant. In this case, the modulus of the filament should be considered, as well as its affect on the filament force $F_{f}$.

Assuming that the helical yarn follows a local sinusoidal path, the effective cross-sectional area of the yarn $\left(A_{h}\right)$ is given by the following equation ${ }^{14}$

$$
A_{h}=\frac{S}{\bar{b}} \times A
$$


Where $S$ is the yarn's actual length, $b$ is the crossing yarn spacing as measured along the $x$-axis (Figure 3 ), and $A$ is the cross-sectional area of the yarn.

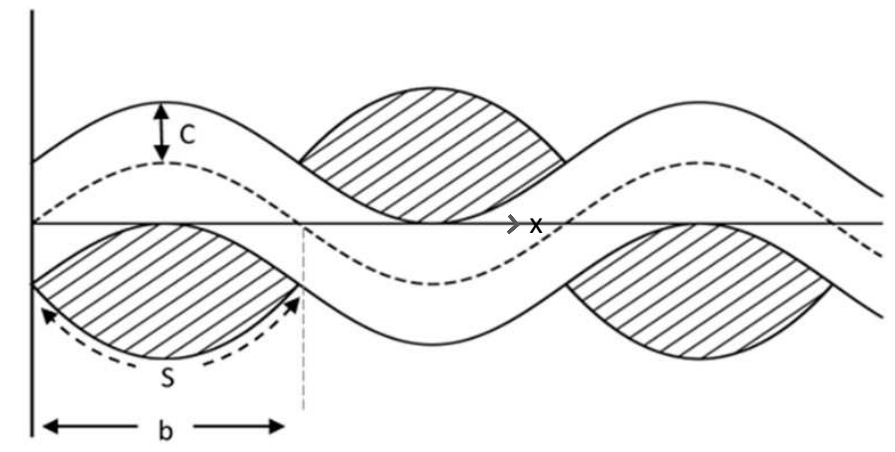

Figure 3. Yarn crossover model for a diamond tubular braid.

The effective cross-sectional area takes into account the ellipsoidal shape as shown in Figure 4 and is expressed in the following equation ${ }^{14}$ :

$$
A_{h \alpha}=\frac{S}{\bar{b}} \times \frac{A}{\cos (\alpha)}
$$




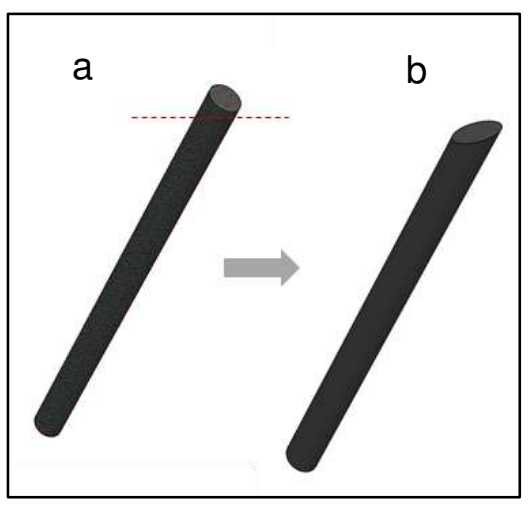

Figure 4. Cross-sectional area (a) of yarn converted to the effective cross-sectional area(b).

The fiber crimp, c, can be related to the ratio between the actual length of the yarn and the crossing yarn spacing as measured along the $\mathrm{x}$-axis ${ }^{19}$ :

$$
c=\frac{S}{\bar{b}}-1
$$

Equations (2.19) and (2.20) give

$$
A_{h \alpha}=\frac{\pi d_{f}^{2}(C+1)}{4 \cos (\alpha)}
$$

In the case of multifilament yarns, the effective cross-sectional area of the braid $\left(\boldsymbol{A}_{\boldsymbol{b}}\right)$ is

$$
A_{b}=\sum_{i=1}^{N} A_{h \alpha_{i}}=N A_{h \alpha}=\frac{N \pi d_{f}^{2}(C+1)}{4 \cos (\alpha)}
$$

Furthermore, the cross-sectional area of the core $\left(A_{c}\right)$ 


$$
A_{c}=\frac{\pi D^{2}}{4}
$$

Therefore, the effective cross-sectional area of tubular braid consisting of the elastic core $\left(A_{b c}\right)$ is:

$$
A_{b c}=\frac{N \pi d_{f}^{2}(C+1)}{4 \cos (\alpha)}+\frac{\pi D^{2}}{4}
$$

Combining equations (2.1), (2.2), (2.4), (2.14), (2.15), (2.23) and (2.24), the following relationship is obtained for the stress:

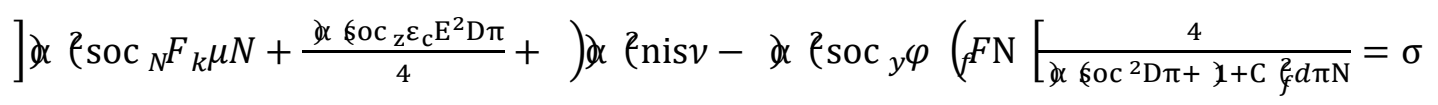

The braiding angle $(\alpha)$ is changing during stretching and can be calculated by the following equation:

$$
\cos (\alpha)=\frac{1+\varepsilon_{z}}{1+\varepsilon_{f}} \cos \left(\alpha_{i}\right)
$$

Hence, all the variables such as the yarn tension $\left(F_{f}\right)$ and normal force $\left(F_{N}\right)$ are included as shown in equations (2.5) and (2.17), respectively.

The solution of the model provides the following stress-strain graph as shown in Figure 5. With a higher braiding angle, the structure, theoretically, could stretch more. The maximum strain is due to the locking state, which is predicted by equation (1.7). 


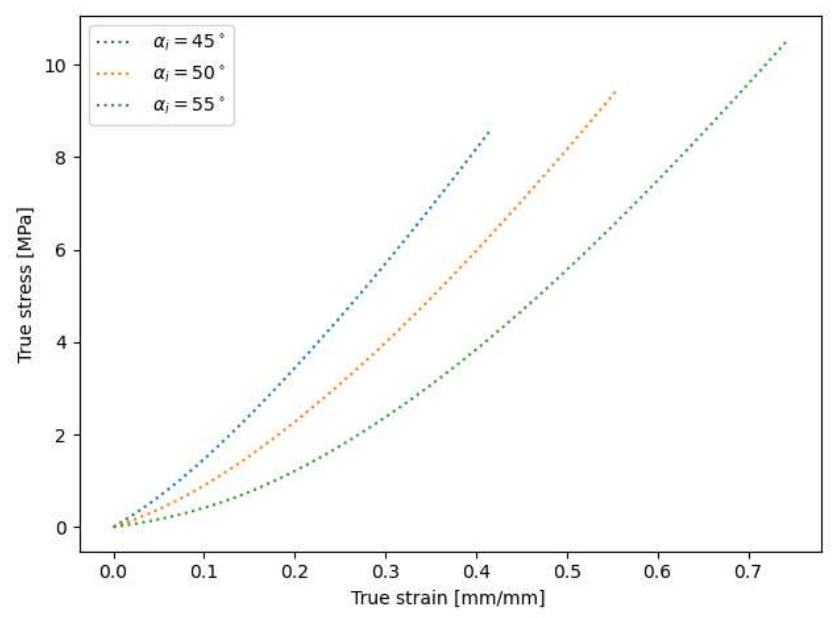

Figure 5. Theoretical true stress-strain curves of braid on core composite for different initial braiding angles.

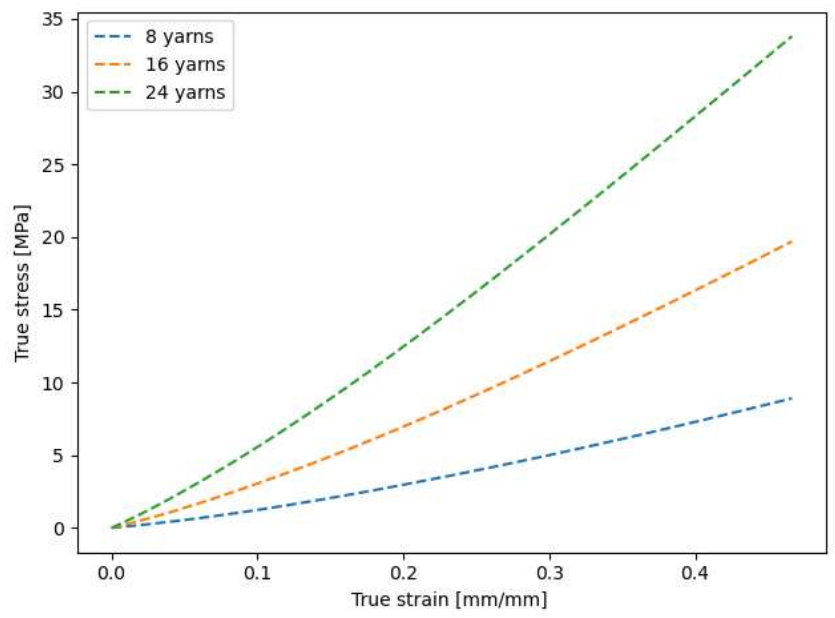

Figure 6. Theoretical true stress-strain curves of braid on core composite for different number of yarns where $\boldsymbol{\alpha}_{\mathbf{i}}=\mathbf{4 7}^{\circ}$.

Figure 5 describes the calculated stress-strain relationship using equation 2.25 . The limit strain for each angle is the locking angle. As is evident, with the increase of the braiding angle from $45^{\circ}$ to $55^{\circ}$, the hybrid structure's strain increases from 0.4 to 0.7 , indicating that the braiding angle is the limiting factor for the 
system's total strain. Figure 6 describes the stress-strain curves for $\alpha_{i}=47^{\circ}$ using different numbers of yarns. As can be noted, with a higher number of yarn, the more significant is the friction force.

\section{Range III:}

In this range, the core squeezes out through the braid; therefore, the braid is locked, and the angle cannot change (decrease) further. Hence, the yarns are in tensile stress, and the yarn modulus of elasticity controls the resulting strain to the point of fiber failure.

\section{Experimental results}

\section{Materials}

For the fibers, CNTYs (Miralon ${ }^{\circledR}$ yarn Nanocomp Technologies, Inc. Merrimack, New Hampshire) was used. The material is a direct-spun untreated single-ply CNTY with 10 tex $(10 \mathrm{~g} / \mathrm{km})$ linear density, $2800 \mathrm{~s}^{*} \mathrm{~cm}^{2} / \mathrm{g}$ specific conductivity, $\sim 0.7 \mathrm{~g} / \mathrm{cm}^{3}$ density and a diameter of $\sim 150$ microns (Nanocomp A-series) ${ }^{23}$. A commercially available silicone rubber cord was used for the core material (High-Temperature soft silicone o-Ring cord stock 0.007", McMaster-CARR)

\section{Fabrication of The Braid}

The braidings were fabricated using a braiding machine (Wardwell Braiding, W6-355, 2018) with 8 or 16 rotating CNTYs $(130 \mu \mathrm{m})$. The braided textile was fabricated on the silicone rubber core. 


\section{Characterization}

The composite tensile load-deformation relationship was studied using a mechanical tester (Instron4466, Instron ${ }^{\circledR}$, Norwood, Massachusetts), with a $2 \mathrm{kN}$ load capacity. The displacement of the grips was used to calculate the strain, assuming a gauge length of $40 \mathrm{~mm}$. The samples were subjected to 15 cycles of stretching/relaxation to predetermined strain values at a rate of $50 \mathrm{~mm} / \mathrm{min}$, while simultaneously measuring the electrical resistance. At least three samples were tested under the same conditions for each loading procedure.

The extension profile is described in Figure 7, where the composite samples were subjected to 15 cycles of stretching/relaxation using strain rates of 50 and $5 \mathrm{~mm} / \mathrm{min}$. At least four specimens were characterized under the same conditions for each result; The composite's (rubber core and the CNTYs) electrical properties were measured using a two-probe electrical resistance device (FLUKE 179 multimeter). The measuring probes were connected by conductive copper foil tape to the braid structure to achieve good contact between the braid and the probes. An insulating layer between the composite/conductive tape and the clamps was used for insulation. The applied voltage was $5 \mathrm{kV}$.

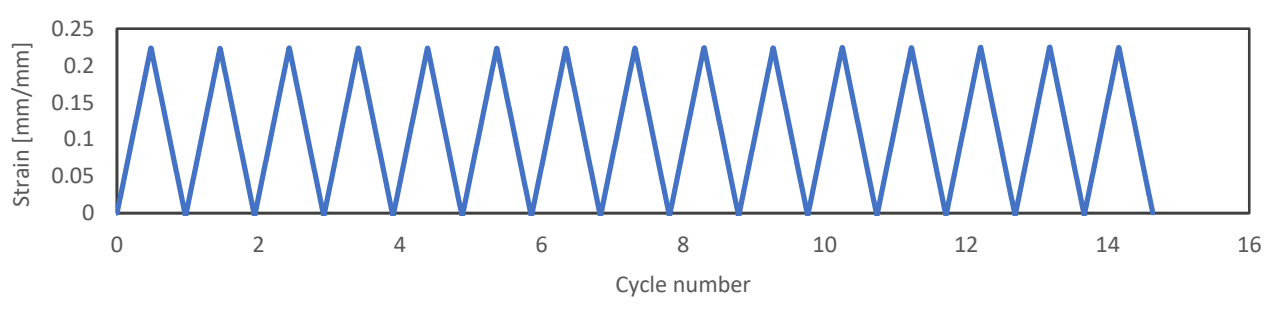

Figure 7. extension profile during the electrical measurements 


\section{Stretching Visualization and Tensile Test}

The composite braid tensile behavior was studied using a Mechanical Tester (4466 Instron machine) with a $2 \mathrm{kN}$ loading capacity. The displacement of the grips was used to calculate the strain, using a gauge length of $40 \mathrm{~mm}$. The elongation rate was studied, and hence $50 \mathrm{~mm} / \mathrm{min}$ extension rate was used. At least five samples were characterized under the same conditions. A high-speed camera was used to follow the composite stretching with simultaneous monitoring of the stress-strain response. A stop watch was used to synchronize between the time in the video and the loading machine's time.

Three failure modes were found as depicted in Figure 8. In failure mode 1 the rubber squeezes out of the braid, in failure mode 2 there is failure of the yarn(s), and in failure mode 3 there is failure of the rubber core.
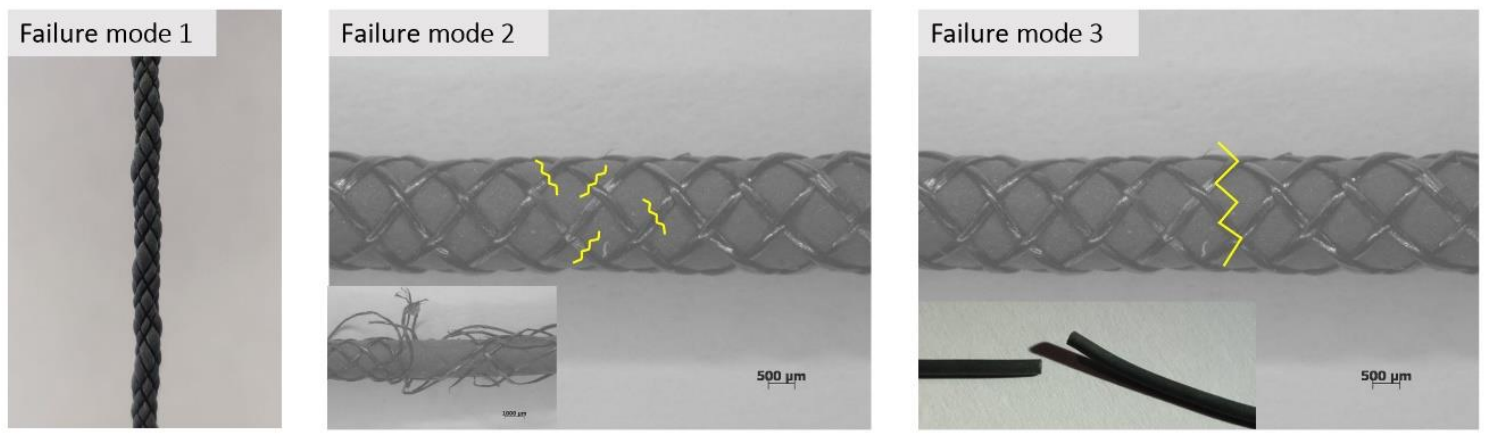

Figure 8: Three failure modes

Figure 9 presents captured photos from the video camera. Figure 9.B shows the start of 'squeezing out'. For $\alpha_{i}=47^{\circ}$; the core started to squeeze out around $\varepsilon \approx 16 \%$, as shown in Figure 10 . The drop in the stress 
during the test indicate the breaking of the yarns during the stretching (Failure mode 2). This supports the assumption that once the 'squeezing out' occurs, the yarns start to stretch.

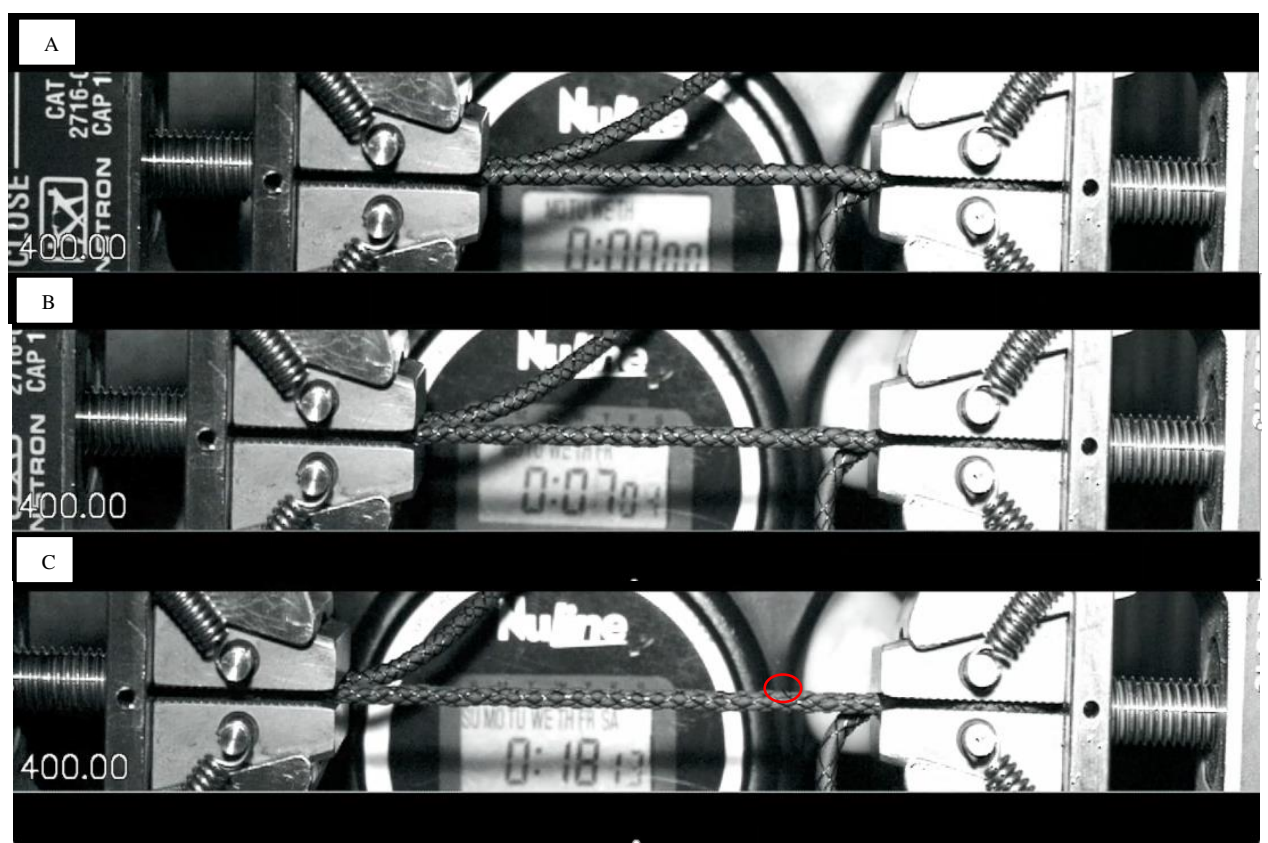

Figure 9. Pictures of different times during the tensile test measurement. (A)- at the beginning Osec, (B) at $7.04 \mathrm{sec}$ - 'squeezing out' (failure mode 1) starts to occur, and (c) $18.13 \mathrm{sec}$ first failure mode 2 appears. 


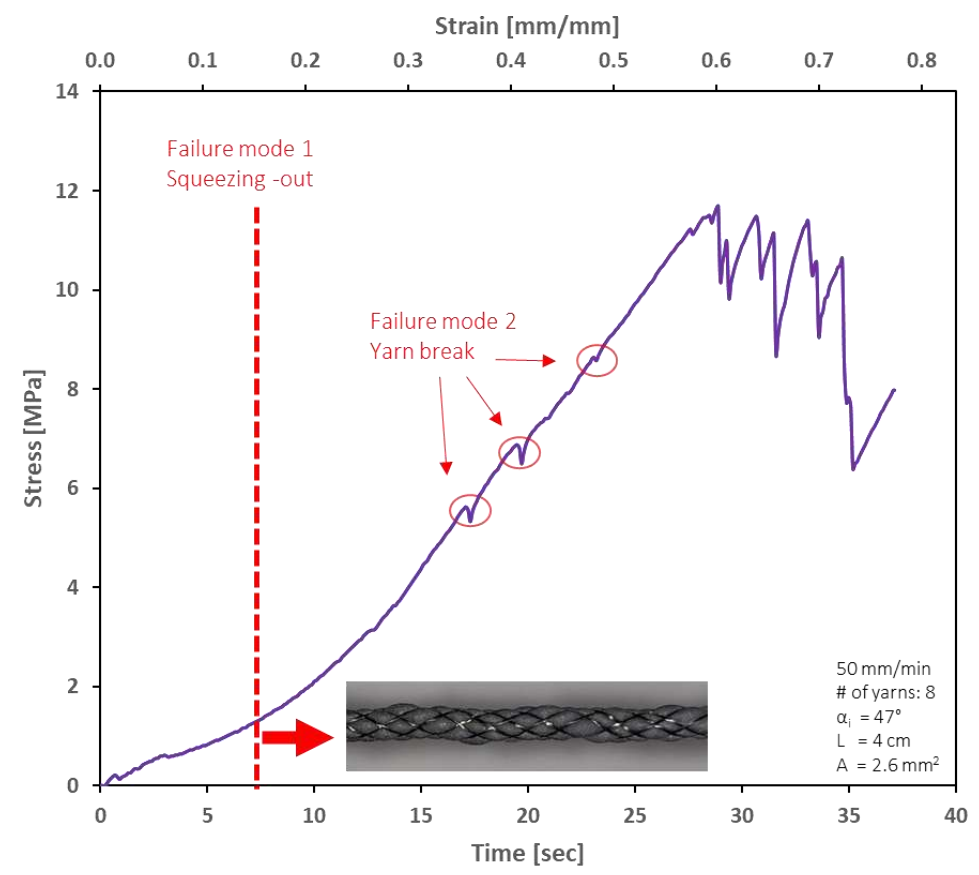

Figure 10. Plot of stress versus time (bottom scale) and strain (upper scale).

Figure 11 depicts the influence of the number of yarns, 8 and 16, braided on the core. In the initial part of the curve, the stress-strain relationship is similar as the rubbery core is dominant. Close to the 'squeeze out' point, significant changes occur because the yarns stretch and their contribution to the stress is significant. 


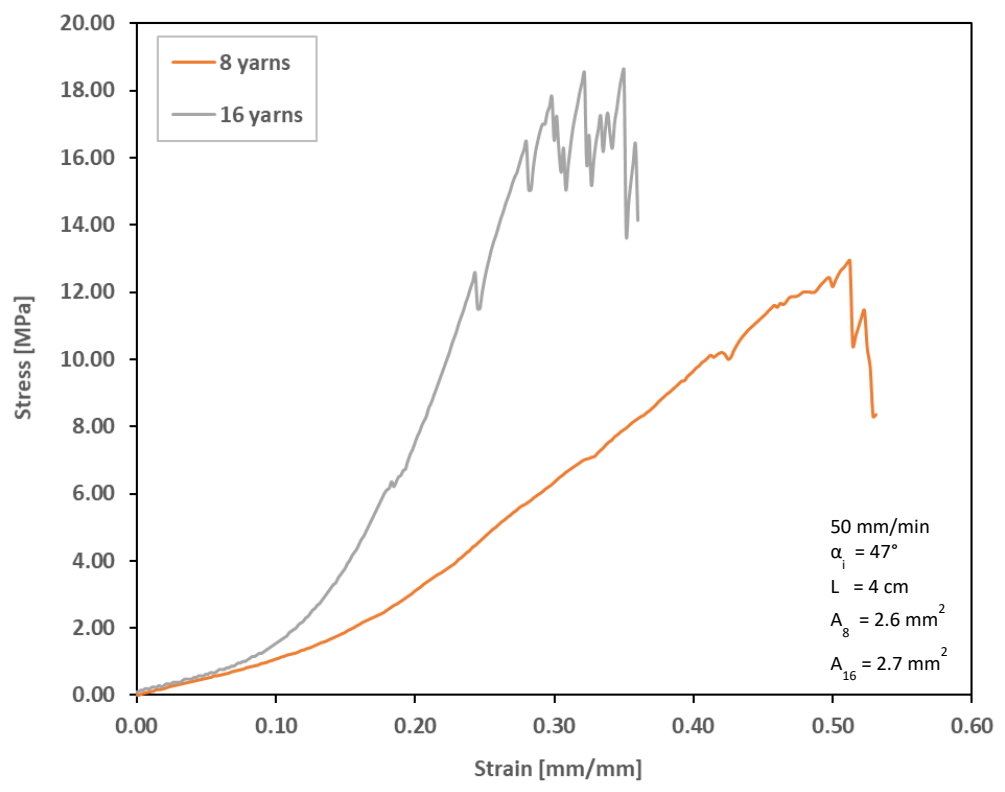

Figure 11. Experimental stress-strain curves of braid on core composite for different number of yarns.

The experimental results are compared to the proposed model (Figure 12). As can be seen, there is a good agreement between the experimental results and the proposed model compared to that of Rawals ${ }^{\prime 19}$ model. This may be attributed to the effect of the friction contribution between the braid and the core. The proposed model is in good agreement with the experimental results up to the point where the 'squeezing out' occurs ( $15 \%$ strain). At this point, the stress of the composite increases significantly due to the stretching of the yarns. Figure 13 displays the simulated stress-strain curves for $\alpha_{i}=47^{\circ}$, applying four different coefficients of friction: $0.7,0.6,0.5,0.001$, and Rawals ${ }^{\prime 19}$ model results. The first 3 are typical friction coefficients for rubber. The difference between the curves (0.001 to 0.5$)$ before failure mode 1 is almost $40 \%$, indicating that the friction cannot be neglected. The results also indicate that when the coefficient friction is almost zero, the proposed model is similar to the Rawals ${ }^{19}$ model. 


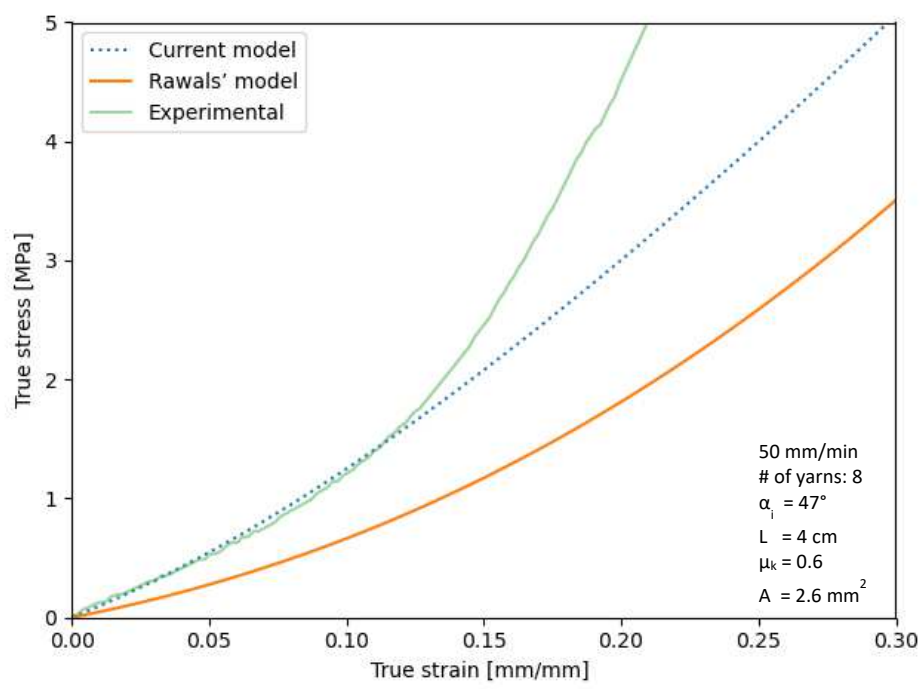

Figure 12. True stress versus true strain for the model, Rawals model, and experimental results $\boldsymbol{\alpha}_{\mathbf{i}}=$ $47^{\circ}$.

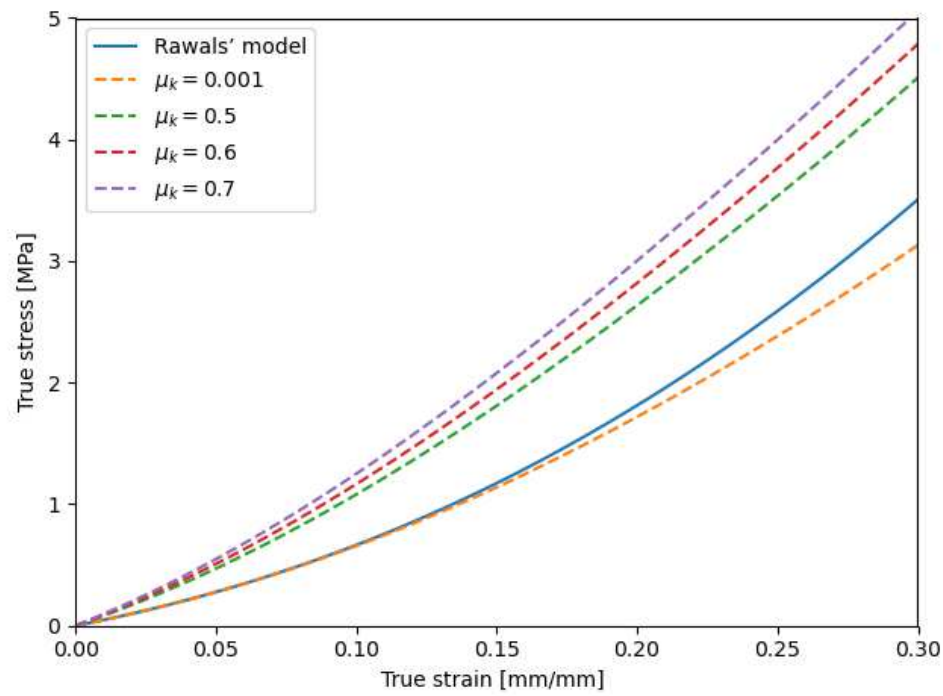

Figure 13. Theoretical true stress versus true strain curves of braid on core composite for different friction coefficients where $\alpha_{i}=47^{\circ}$.

An apparent friction coefficient was proposed to include the effect of the squeeze-out phenomena, as well as changes in the friction coefficient due to stretching. This coefficient considers the change in the friction coefficient during the stretch in part due to changes in contact area and pressure between the yarn and core and is a function of the strain. This apparent friction coefficient replaces $\mu_{k}$ in equation (2.15). At low strain, there is no contact between the braid and the core. In this region it is expected that the coefficient will be close to zero. As the strain 
increases, the braid contacts the core, contact area and pressure increase and the friction coefficient increases linearly. As squeezing out begins to occur the core prevents the yarns from sliding. The friction coefficient is determined using an iterative scheme to minimize the difference between the experimental results and the calculated values by the proposed model. Figure 14 shows that the calculated apparent friction coefficient increases linearly with stretching of the composite braid. This linear relationship indicates that in mode I region, where the braid and the core are not in contact, the apparent friction coefficient is small. As the stretching is increased and the squeezing out phenomena takes place, which prevents the movement of the yarn, the apparent friction coefficient is relative large. Figure 15 shows a good agreement between the theoretical calculation and the experimental results, once the concept of the variable friction coefficient is used.

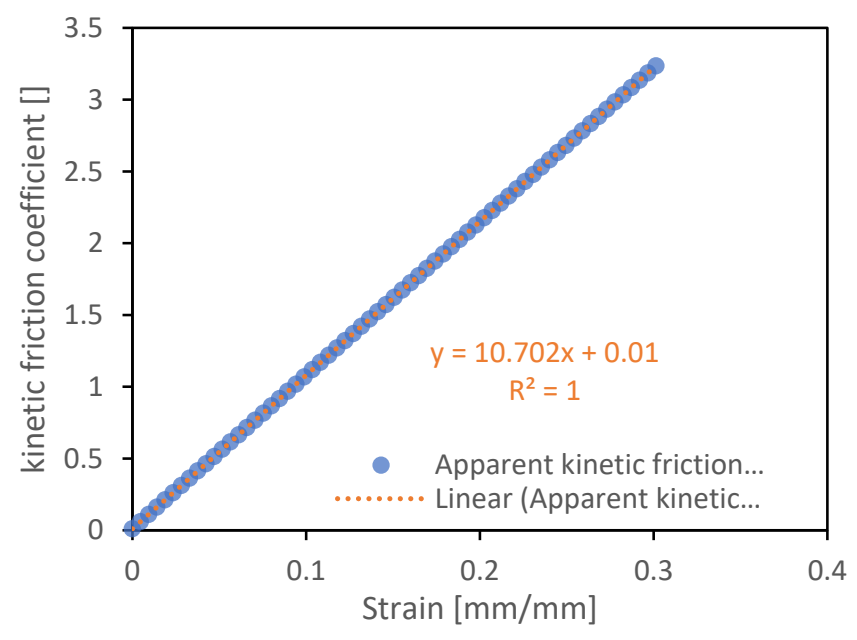

Figure 14. The relationship of the apparent kinetic friction coefficient with strain. 


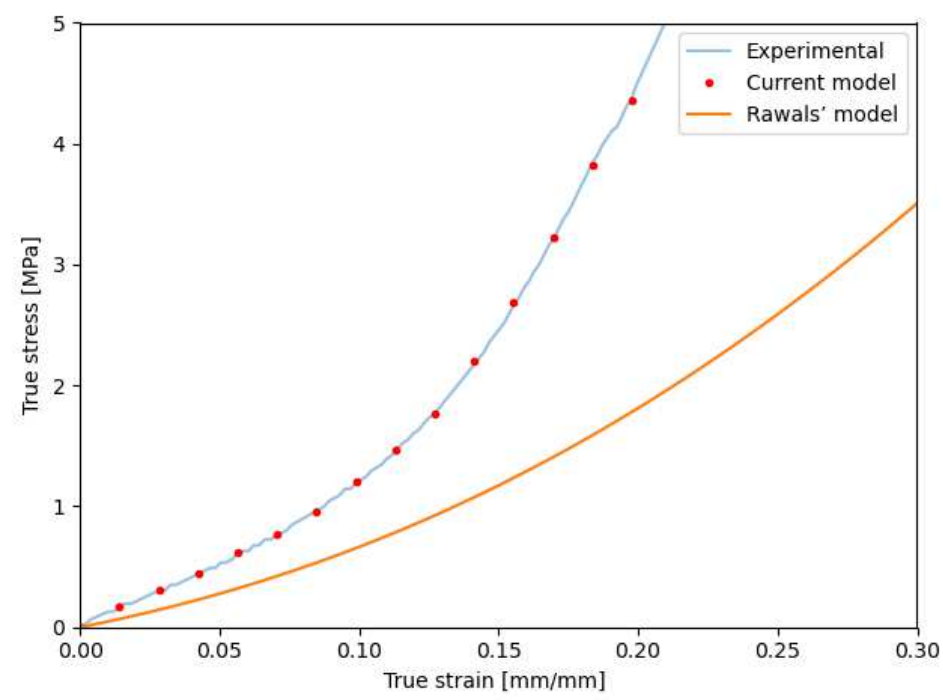

Figure 15. True stress versus true strain for the model with the apparent kinetic friction coefficient, Rawals model, and experimental results $\alpha_{\mathbf{i}}=\mathbf{4 7}^{\circ}$.

\section{Electrical Measurements During Tensile Testing}

The electrical resistance of the braid on the core was evaluated simultaneously with the stress-strain measurements during extension-relaxation cycles up to $22 \%$, which was above the 'squeezing out' strain. The following equation was used to calculate the resistance

$$
R=\frac{\rho L}{A}
$$

Where $R$ is the resistance, $\rho$ is the resistivity, $L$ is the length, and $A$ is the total cross-section. Thus, the resistance ratio between 8 and 16 yarns is:

$$
\frac{R_{8}}{R_{16}}=\frac{\frac{\rho L}{8 A_{\text {yarn }}}}{\frac{\rho L}{16 \text { A }_{\text {yarn }}}}=2 \quad \rightarrow \quad R_{8}=2 R_{16}
$$


The resistance change during deformation was measured when the braided composite was stretched to $22 \%$. As can be seen in Figure16, almost no changes were noticed in the relative resistance $\left(R-R_{0} / R_{\text {that }}\right)$ for both rates and the number of yarns. The experimental stability in the electrical resistance indicates that the braided composite can be used for interconnecting electronic devices (Figure 16 a \& b). Figure 16 c \& d shows that the ratio between the 16 and 8 braided yarns is close to the theoretical value of 2 (see equation 3.2). Furthermore, during 15 cycles, the composite shows stable electrical performance without a change in resistance.
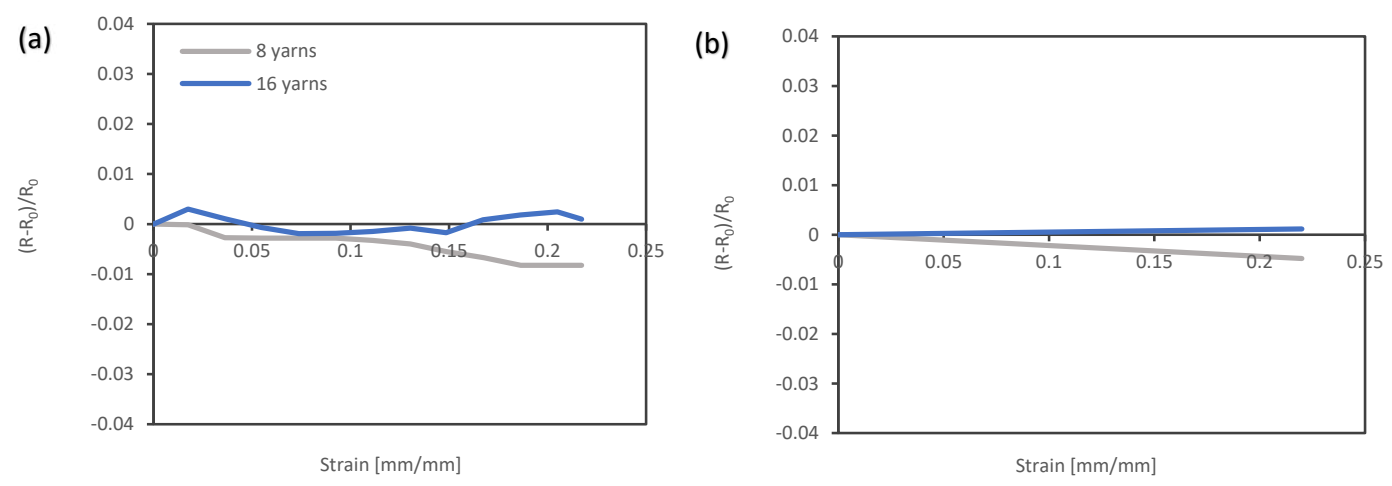

(c)

(d)
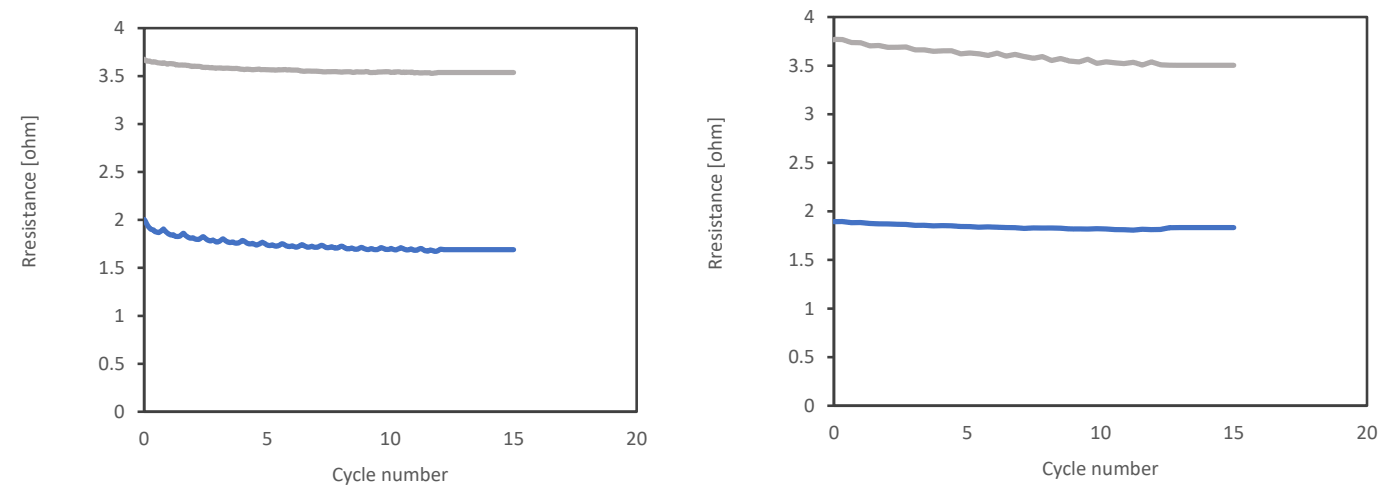

Figure 16. Measurement of the electrical resistance change of the braided composite at a strain of $22 \%$ at a rate of 5 (a) and $50 \mathrm{~mm} / \mathrm{min}(\mathrm{b})$; measurement of the resistance during 15 cycles to $22 \%$ strain with (c) and (d) with a rate of 5 and $50 \mathrm{~mm} / \mathrm{min}$, respectively. 
The composite's resistivities and conductivities were calculated based on its resistance value, length, and cross-sectional area; the average values are shown in Table 2 and Figure 17. For comparison copper possesses resistivity and conductivity of $1.6 \times 10^{-6}[\mathrm{ohm} * \mathrm{~cm}]$ and $5.9 \times 10^{5}\left[\mathrm{~S}^{*} \mathrm{~cm}^{-1}\right]$, respectively. The calculations are approximate and do not consider the changes in the cross-section of the yarns during extension. Therefore, it is reasonable for there to be a slight difference between the different numbers of yarns.

Table 2: Average Value of The Resistivity and Conductivity for 8 and 16 Yarns

\begin{tabular}{c|ccc} 
& Resistivity $\left[\mathrm{ohm} \mathrm{Cm}^{*} \mathrm{~cm}\right.$ & Conductivity $\left[\mathrm{S}^{*} \mathrm{~cm}^{-1}\right]$ & SD $\left[\mathrm{ohm} \mathrm{cm}^{*}\right]$ \\
\hline 8 yarns & $8.4 \times 10^{-4}$ & $1.2 \times 10^{+3}$ & $6.8 \times 10^{-6}$ \\
16 yarns & $9.7 \times 10^{-4}$ & $1.0 \times 10^{+3}$ & $6.7 \times 10^{-7}$
\end{tabular}

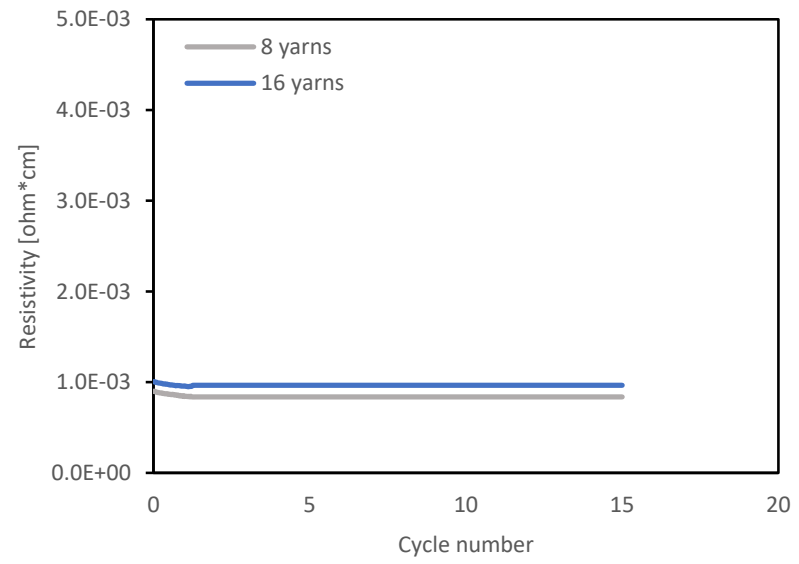

Figure 17. Electrical resistivity of the braided composite at strain of $22 \%$ at a rate of $50 \mathrm{~mm} / \mathrm{min}$ during 15 cycles.

\section{Conclusions}

The tensile stress-strain behavior of rigid braid on elastic core composite was modeled and analyzed. Simulated results demonstrated that as the braiding angle increased, the strain of the composite increased as well. 
The structure deforms in three possible modes that relate to the contact between the braid and the core and the locking of the braid yarns due to the jammed state.

Moreover, friction between the braid and the core significantly influences the hybrid structure's stress-strain behavior and adds accuracy to the Rawals ${ }^{19}$ model. As previously presented $^{15}$, the combination of the braid and core provide a nonlinear tensile response, although all components are considered linearly elastic materials.

An apparent friction coefficient was proposed to account for the rubber core and braid yarn interaction and friction. Consideration of this coefficient in the model leads to improvement of the model's accuracy and allows to model the whole three modes proposed.

Furthermore, the material's resistivity seems to have no significant change even after 15 cycles. The composite's electrical resistivity and conductivity are $9.05 \times 10^{-4}[\mathrm{ohm} * \mathrm{~cm}]$ and $1.1 \times 10^{3}\left[\mathrm{~S}^{*} \mathrm{~cm}^{-1}\right]$, respectively, and considered to be in the range between conductive to semi-conductive material.

Since the phenomena of 'squeezing out' limited the strain that could achieve, different configurations should be considered for future work. 


\section{List of abbreviations}

carbon nanotube yarns (CNTYs)

\section{Declarations}

\section{Availability of data and materials:}

The datasets used and/or analysed during the current study are available from the corresponding author on reasonable request.

Competing interests:

The authors declare that they have no competing interests.

\section{Funding:}

The David and Frances Pernick funded a graduate student to preform the experiment and modeling and write the manuscript.

\section{Authors' contributions:}

AV SK HD JM contributed to the design of the work interpretation of data and revision of the manuscript. AV was involved in the acquisition, analysis, the creation of new software used in the work and manuscript draft.

\section{Acknowledgements:}

The authors would like to knowledge Nanocomp for supplied that CNTYs, Wardwell braiding for the braiding machine. 


\section{References}

1. S.H. Ko, D. Lee ZW. Flexible and Stretchable Electronics. MDPI; 2018.

2. Sim K, Rao Z, Ershad F, Yu C. Rubbery Electronics Fully Made of Stretchable Elastomeric Electronic Materials. Adv Mater. Published online 2020. doi:10.1002/adma.201902417

3. Kim DC, Shim HJ, Lee W, Koo JH, Kim DH. Material-Based Approaches for the Fabrication of Stretchable Electronics. Adv Mater. Published online 2020. doi:10.1002/adma.201902743

4. Hong S, Lee S, Kim DH. Materials and Design Strategies of Stretchable Electrodes for Electronic Skin and its Applications. Proc IEEE. 2019;107(10):2185-2197. doi:10.1109/JPROC.2019.2909666

5. Hu X, Dou Y, Li J, Liu Z. Buckled Structures: Fabrication and Applications in Wearable Electronics. Small. 2019;15(32):1804805. doi:10.1002/smll.201804805

6. Huang S, Liu Y, Zhao Y, Ren Z, Guo CF. Flexible Electronics: Stretchable Electrodes and Their Future. Adv Funct Mater. 2019;29(6):1805924. doi:10.1002/adfm.201805924

7. Larson C, Peele B, Li S, et al. Highly stretchable electroluminescent skin for optical signaling and tactile sensing. Science (80- ). 2016;351(6277):1071-1074. doi:10.1126/science.aac5082

8. Choi S, Han SI, Kim D, Hyeon T, Kim DH. High-performance stretchable conductive nanocomposites: Materials, processes, and device applications. Chem Soc Rev. 2019;48(6):15661595. doi:10.1039/c8cs00706c

9. Chen H, Bai L, Li T, et al. Wearable and robust triboelectric nanogenerator based on crumpled gold films. Nano Energy. Published online 2018. doi:10.1016/j.nanoen.2018.01.032

10. Wang J, Lin MF, Park S, Lee PS. Deformable conductors for human-machine interface. Mater Today. Published online 2018. doi:10.1016/j.mattod.2017.12.006

11. Dabiryan H, Johari MS. Analysis of the tensile behavior of tubular braids using energy method, part I: theoretical analysis. J Text Inst. 2016;107(5):553-561.

doi:10.1080/00405000.2015.1046309 
12. Schreiber F, Theelen K, Schulte Südhoff E, Lee HY, Ko FK, Gries T. 3D-hexagonal braiding:

Possibilities in near-net shape preform production for lightweight and medical applications. ICCM Int Conf Compos Mater. Published online 2011:2-5.

13. Rawal A, Saraswat $\mathrm{H}$, Sibal A. Tensile response of braided structures: A review. Text Res J. 2015;85(19):2083-2096. doi:10.1177/0040517515576331

14. Phoenix SL. Mechanical Response of a Tubular Braided Cable with an Elastic Core. Text Res J. 1978;48(2):81-91. doi:10.1177/004051757804800204

15. Abbott GM. Force-Extension Behavior of Helically Wrapped Elastic Core Yarns. Text Res J. 1984;54(3):204-209. doi:10.1177/004051758405400311

16. Hopper RH, Wallace Grant J, Popper P. Mechanics of a Hybrid Circular Braid with an Elastic Core. Text Res J. Published online 1995. doi:10.1177/004051759506501202

17. Rawal A, Saraswat H, Kumar R. Tensile response of tubular braids with an elastic core. Compos Part A Appl Sci Manuf. 2013;47(1):150-155. doi:10.1016/j.compositesa.2012.12.006

18. Harte AM, Fleck NA. On the mechanics of braided composites in tension. Eur J Mech A/Solids. Published online 2000. doi:10.1016/S0997-7538(99)00164-3

19. Rawal A, Saraswat H, Kumar R. Tensile response of tubular braids with an elastic core. Compos Part A Appl Sci Manuf. Published online 2013. doi:10.1016/j.compositesa.2012.12.006

20. Gu B. Prediction of the uniaxial tensile curve of 4-step 3-dimensional braided preform. Compos Struct. Published online 2004. doi:10.1016/j.compstruct.2003.08.009

21. Rawal A, Sibal A, Saraswat H, Khan SQ. Topologically controlled tensile behaviour of braided prostheses for anterior cruciate ligaments. J Mech Behav Biomed Mater. 2016;57:359-364. doi:10.1016/j.jmbbm.2016.01.033

22. Rawal A, Kumar R, Saraswat H. Tensile mechanics of braided sutures. Text Res J. 2012;82(16):1703-1710. doi:10.1177/0040517512445340 
23. MIRALON ${ }^{\circledR}$ :: Huntsman Corporation (HUN). Accessed October 5, 2021.

https://www.huntsman.com/products/detail/344/miralon/yarn 


\section{Appendix 1- STRETCHABLE CONDUCTIVE MATERIALS BASED ON BRAIDED CNT YARNS ON ELASTOMERIC CORE}

The diameter for the braid (d) is presented in the following equation, based on geometric relations (Figure 1):

$$
\mathrm{d}=\frac{L \sin (\alpha)}{\pi}=\frac{h}{\pi} \tan (\alpha)=\frac{h_{0}\left(1+\varepsilon_{z}\right)}{\pi} \tan (\alpha)=\frac{h_{0}\left(1+\varepsilon_{z}\right)}{\pi}\left(\frac{1}{\cos ^{2}(\alpha)}-1\right)^{0.5}
$$

Where, $L$ is the length of the yarn, $z$ and $z_{0}$ are the axial length of the helix and the axial length of the helix at zero levels of axial strain, respectively.
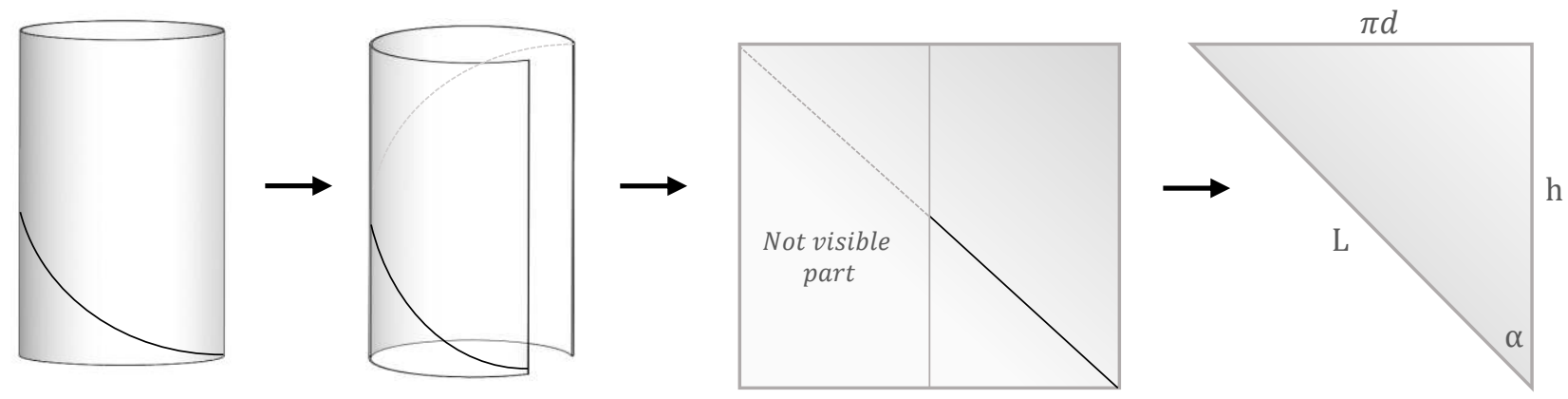

Figure i.18. Geometric relation of a helix

The geometric expression for $\cos (\alpha)$ is given in the next equation:

$$
\cos (\alpha)=\frac{h}{L}=\frac{h_{0}\left(1+\varepsilon_{z}\right)}{L_{0}\left(1+\varepsilon_{f}\right)}=\frac{1+\varepsilon_{z}}{1+\varepsilon_{f}} \cos \left(\alpha_{i}\right)
$$

Where $\alpha_{i}$ is the initial braiding angle and $\varepsilon_{f}$ is the yarn strain. For this case, we assume that the yarn strain is zero, therefore

$$
\cos (\alpha)=\left(1+\varepsilon_{z}\right) \cos \left(\alpha_{i}\right)
$$


Combining equations i.1 and i.3 provide

$$
\mathrm{d}=\frac{\mathrm{h}_{0}}{\pi}\left(\frac{1}{\cos ^{2}\left(\alpha_{i}\right)}-\left(1+\varepsilon_{\mathrm{z}}\right)^{2}\right)^{0.5}
$$

Using the geometry relationship as shown in the following equation:

$$
\tan \left(\alpha_{i}\right)=\frac{\pi \mathrm{d}_{0}}{\mathrm{~h}_{0}}
$$

Gives:

$$
\frac{\mathrm{d}}{d_{0}}=\frac{1}{\tan \left(\alpha_{i}\right)}\left(\frac{1}{\cos ^{2}\left(\alpha_{i}\right)}-\left(1+\varepsilon_{z}\right)^{2}\right)^{0.5}
$$

